\title{
Intelligent wireless sensor network for wildfire detection
}

\author{
A. Ko ${ }^{1}$, N. M. Y. Lee ${ }^{1}$, R. P. S. Sham ${ }^{2}$, C. M. So ${ }^{2} \&$ S. C. F. Kwok ${ }^{2}$ \\ ${ }^{1}$ Intelligent Systems Laboratory, \\ The University of Hong Kong, Hong Kong \\ ${ }^{2}$ Insight Robotics Limited Company, Hong Kong
}

\begin{abstract}
Applications of Wireless Sensor Network (WSN) can be found anywhere from small manufacturing plants to major military operations. The inherited scalability and robustness of WSN make them an indispensible component in systems designed to monitor and communicate field data over large areas. While rural fire detection systems can most certainly benefit from these innate features of WSN, there are various technical difficulties to overcome to fully utilize this technology. This paper describes an intelligent wildfire detection system that can be deployed to a station in the middle of rural forests to monitor abnormal heat occurrences and report to duty officers outside of the forest through the internet or satellite. The intelligent wildfire detection system consists of thermal cameras and WSN can analyse feedback from the two sources to minimize false alarm rate without scarifying reliability. The system has been developed into prototype and installed in Guangdong, China. The system has obtained success in a largescale field test in partnership with the Guangdong Academy of Forestry. After the final evaluation by Provincial Departments in April, the system will be officially adopted by the Guangdong Province and be widely deployed in the province. The purpose of this paper is to present the technical design of the system as well as challenges in the integration and administration of advanced technology in a traditionally human-based, labour-intense system.

Keywords: wildfire detection, search and rescue robots, wireless sensor networks, computer vision, environmental protection, thermal camera, artificial intelligence.
\end{abstract}




\section{Introduction}

Forests are invaluable assets to mankind. They contribute to the health of our global environment by converting carbon dioxide into oxygen, stabilizing the climate in general, and regulating the water cycle by absorbing and redistributing rainwater to lives in and around them. In 2010, there were approximately $44,682,000 \mathrm{~km}^{2}$ of forest on earth, accounting for around $30 \%$ of the Earth's total land area [1]. Unfortunately, this number is diminishing every year due to recurring disturbances, such as fire, illegal logging and mining, and recurrent cycle of large, damaging natural fires. In an effort to prevent more loss of forest, an intelligent wildfire detection system based on wireless sensor network, supported by computer vision is designed and tested in Guangdong, China.

This paper describes the design and testing of an integrated wildfire detection system deployed in China. The system has three sub-systems, cameras, wireless sensor network (WSN), and mobile sub-systems. These sub-systems were designed to support and verify data produced independently to increase reliability and minimize false alarm rate. The camera sub-system combines and analyzes high-resolution images obtained from Short Wave Infrared (SWIR) cameras and thermal cameras to detect fire in a forest from multiple locations. The WSN connects sensor nodes placed in strategic locations throughout the forest provides feedback on temperature, humidity, wind velocity and other local information. The mobile sub-system with necessary manoeuvrability offers verifiable data not obtainable through the static sub-systems. While the mobile sub-system is at prototype stage, this paper will focus on reporting the camera and WSN sub-systems. The WSN forms the backbone of the system in transmitting control signals and field data among the sensors and with the control console. The control console is the powerhouse that integrates and analyses all data. The control console is built around the General Suppressive Control Framework (GSCF) developed by Ko et al. [2] based on artificial immune systems (AIS) [3]. AIS are artificial intelligence systems that mimic the function of the immune system of humans [4]. The advantage of this immune based system is its ability to adapt to unforeseen conditions and generate optimal solutions based on previous experience.

The strength and novelty of this wildfire detection system is in the combined use of vision and sensory data to improve system reliability and efficiency. While WSN can convey field data across large plane and over irregular terrain with high reliability, system cost increases with field dimension and terrain complexity. Camera images, on the other hand, can cover much more area per unit area; hence, system cost remains low for large forest. However, fix-location cameras are good at monitoring large areas with little terrain variations, to monitor valleys and hilly terrains, additional cameras need to be installed, which adds to the system's cost. The hybrid system, as the one presented in this paper, can use cameras as a primary monitoring system to cover the majority of the forest and use WSN to cover irregular terrain. The method utilizes the strength of cameras and WSN to create a reliable and cost effective system. 


\section{Wildfire prevention in China}

Forest biodiversity may be the richest of all terrestrial ecosystems; its plants and animals provide extracts for drugs used against cancer. Forests contain many potential sources of cures that are yet to be discovered. According to World Bank estimates, more than 1 billion people depend on forests for their livelihood. Global forest products traded internationally are estimated at US\$327 billion per year [5]. While forests can cover millions of square meters of land across multiple provinces and countries (e.g. Taiga and Amazon), each state covered by the "same forest" may have different forest protection policy, regulation, technical standard, response capacity, and not to mention climate. Early forest fire detection systems for such applications must be able to adapt to these natural and human factors in order to effectively mitigate damage at the earliest time.

Recognizing the importance of forests to its economy and environment, the Chinese Government released the "China's 12 ${ }^{\text {th }}$ Five-Year Plan" (2011-15) with a sub-section dedicated to forest fire prevention. Section 5.1.15, titled "Forest Fire Prevention" in the document stressed the immediacy to acquire and deploy modern wildfire detection and monitoring systems to improve overall efficiency. The document further identified 67 high-risk forests across the nation and restated the adverse effect of wildfire may have on surrounding provinces. These high-risk areas are divided into class I and Class II, they include provincial forests, national and provincial protected forests, urban forests, the BeijingZhuhai Expressway, the Shenzhen-Shantou Highway, Meihe Highway and Guangdong Jiangxi Highway. These areas will be among the first to adapt modern fire detection and monitoring systems under the direction of the "China's $12^{\text {th }}$ Five-Year Plan." In addition to China, many countries are already implementing new forest protection policies and automated systems to improve efficiency and reliability in detecting forest fire. On this vital front, a reliable automated system is needed to address the problem.

\subsection{Conventional wildfire detection methods}

Three methods are commonly used for detecting forest fires, lookout towers, visual reports and satellite imagery systems. While the first two methods were employed since the first day human wanted to prevent forest fire, each of these three methods has its shortcomings (see Table 1). Compared with the traditional methods (i.e. employ a supervisor to look at the dozens of camera constantly), the cost and operating budget of our proposed system is more effective and also more economically viable. Shorter fire detection time means less damage to the state, environment and public health.

\section{Intelligent wildfire detection system}

The Intelligent Wildfire Detection System was designed to provide a 24-hour wildfire monitoring service to protect precious forests to ensure their sustainable existence. The system can detect fires as small as a few square meters in open 
Table 1: $\quad$ Traditional wildfire detection methods and their disadvantages.

Manned Lookout Towers
-Rely heavily on human
judgment
-Human vision can be
affected by weather,
time, disease, and
psychological factors
-Operation cost is
rapidly increasing,
even in developing
countries
-Difficult to supply
remote lookout
towers

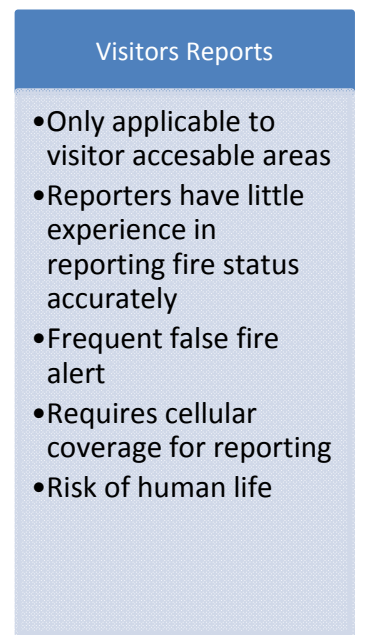

Satellite Imagery Systems
-Image refresh rate is
- High operation cost
-Difficult to analyze
manually
- Can only identify
large-scale Forest fires
- Unable to provide
early and small-scale
fires alert
- Unable to issue
evacuation warnings
to the visitors
immediately

area and can provide accurate, near to real-time images for manual and computer analysis [6]. The wildfire detection system can be configured and deployed in rural and urban areas to continuously acquire and feedback reliable sensory and visual data through its robust wireless network. Designed to suit different field and mission requirement, the module-based system is highly flexible, energy efficient, rugged, and scalable. The system can be easily configured to detect and report wildfire under different climate, terrain, plant types, and other influencing factors within a user-specified period.

Forests can have very diverse geographic features. Some forests are massive in size and remote in distance, others maybe smaller and scatter across urban and rural terrains. This variation imposes great challenge to cost effectively install and maintain manned watchtowers and keep their operators adequately supplied. The Computer Vision Based Wildfire Detection System is designed to resolve this problem by applying advanced artificial intelligence vision analyses technology to eliminate human presence in watchtowers. By moving operators from watchtowers to easily accessible control centers, supply and maintenance cost are significantly lowered, while efficiency and reliability are inversely increased.

The Computer Vision Based Wildfire Detection System consists of infrared thermal cameras, video cameras, high-definition Pan-Tilt-Zoom (PTZ) platform and high-precision computer vision systems. General appearance of the installed system is shown in Figure 1.

As it is, there are several operational steps with regards to the system. Firstly, the system employs intelligent algorithms to identify suspected fire sources in the cameras' visible range. Based on camera images and build-in 3D maps of the region, the system can estimate coordinates of suspected fire sources. When the coordinates are identified, data will be transmitted to the control system and a 


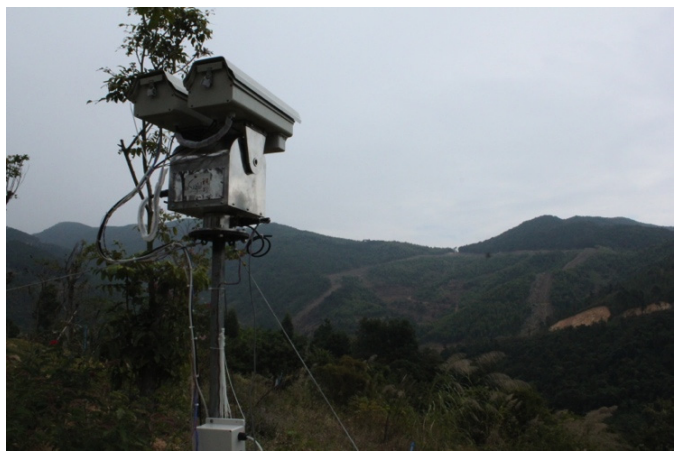

Figure 1: The cameras installed on a weather tight pan-tilt platform.

fire alert will be released. Operators at control centres and relevant authorities will receive instantaneous notification on their computer screens.

The vision system can combine images obtained from the infrared thermal camera (see Figure 2) and visible light camera to produce analyzable computer image. This image provided to operators along with the fire alert and feedback from the sensor network enable operators to have a holistic understanding of the field. The computer system at the console using an artificial intelligence algorithm can make immediate advice to the operator based on current and past data.

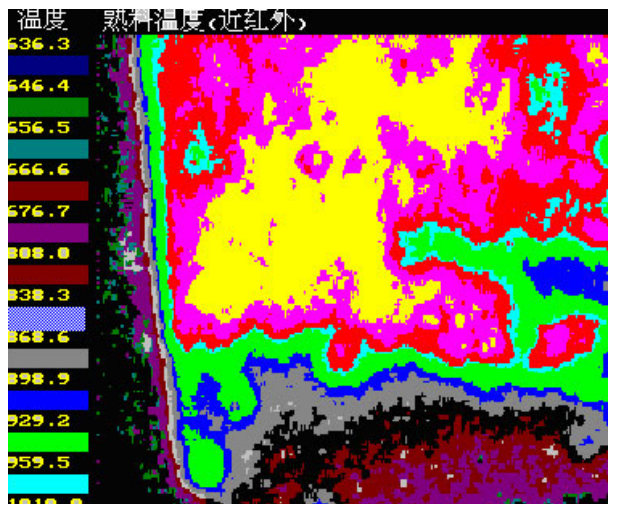

Figure 2: $\quad$ Image obtained from infrared thermal camera.

The artificial intelligence algorithm used is built on the General Suppression Control Framework (GSCF). The GSCF is based around the analogy of suppression hypothesis in the discrimination theory [7]. When a T-cell receptor binds to a peptide with high affinity presented by an APC (Antigen Presenting Cells), the T-cell recognized the antigen become mature and it has to decide whether to attack the antigen aggressively or to tolerate it in peace. An important decision factor is the local environment within which the T-cell resides. The present of inflammatory cytokine molecules such as interferon-gamma (INF- $\gamma$ ) 
in the environment tend to elicit aggressive behaviors of T-cells, whereas the anti-inflammatory cytokines like IL-4 and IL-10 tend to suppress such behavior by blocking the signaling of aggression. In brief, a T-cell matured after recognizing an antigen does not start killing unless the environment also contains encouraging factors for doing so. In addition, after a mature T-cell developed a behavior, it will emit humoral signals to convert others to join. The mechanism was applied to control a 7-link modular robot arm [8], search and rescue robots [2], self-balancing robots [9], and other intelligent systems that need to deal with abnormality in operation. The wildfire detection system takes advantage of the GSCF to learn from past experience and determine if there is a new fire. Table 2 depicts the action flow from the identification of a possible fire to the issue of an alert.

Table 2: Action flow of the computer vision based wildfire detection system.

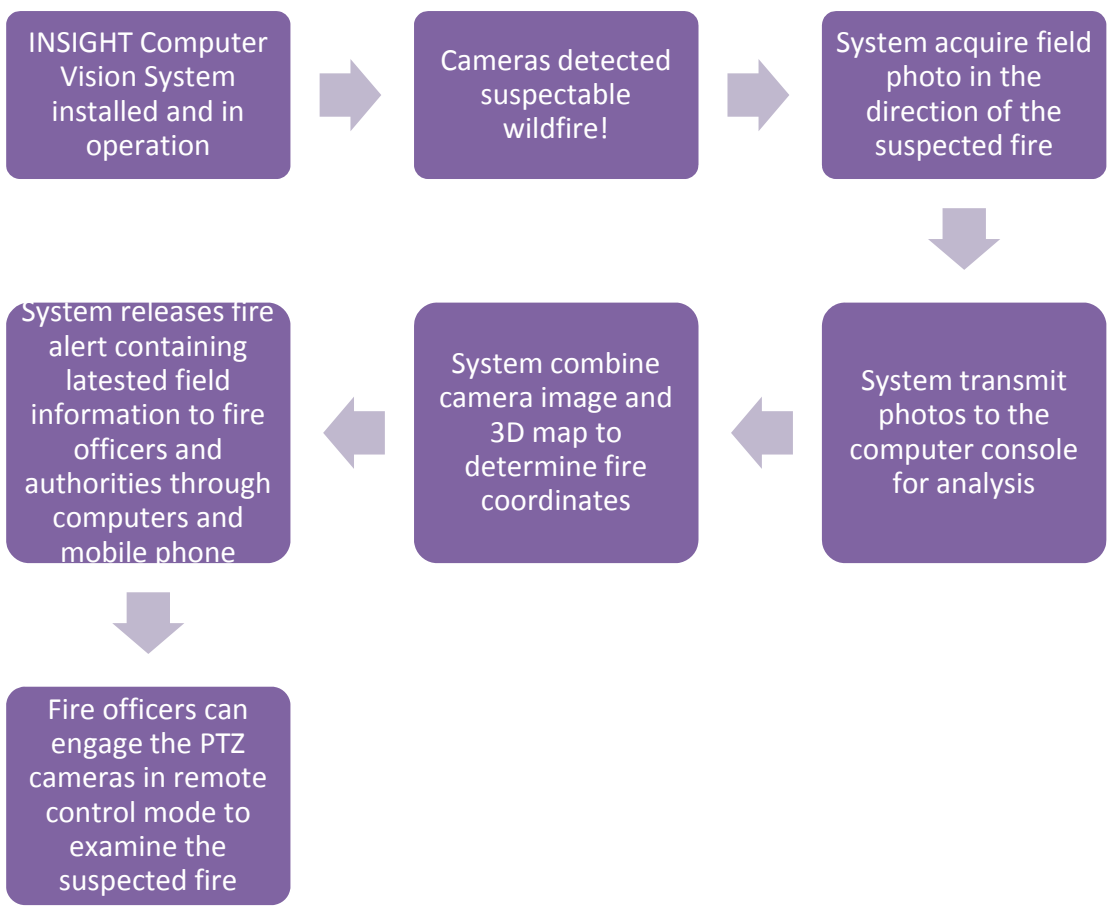

\subsection{System design}

The Computer Vision Based Wildfire Detection System integrates the best qualities of infrared thermal camera and a visible camera to generate reliable images under different environments and adverse weather conditions. Designed with flexibility in mind, all key components and peripheral in the system share identical communication protocol and are supported under a module-based 
system. The result is an integrated system that is reliable, robust and easy to maintain. The system is also equipped with a remote and automatic monitoring system. Whether the system is deployed in a forest 16 hours by road from the nearest town or right behind a rural town, the automated system can provide instantaneous notifications to Internet connected computers and mobile devices, and cellular phones. The system can detect fire as small as $1 \mathrm{~m} \times 2 \mathrm{~m}$ from a distance of $3 \mathrm{~km}$. The sensor network can detect a fire as small as $16 \mathrm{~m}^{2}$ within 30 minutes. The best commercially available satellite can only detect fire larger than $500 \mathrm{~m} \times 500 \mathrm{~m}$ at a refresh rate between 3-6 hours, this new system is significantly faster and more sensitive.

\subsection{Wireless sensor network}

Wireless Sensor Network applied in many different fields including, medical [10], civil [11], and environment research [12], has demonstrated its value in conveying data over large area with high level of power efficiency. Design of the WSN for the wildfire system was based on a previously developed low-cost autonomous search and rescue robot tracking system (Figure 3) presented in [2].

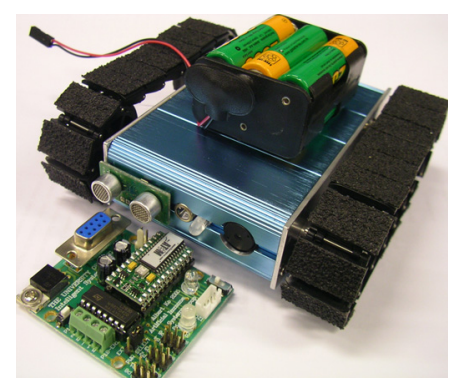

Figure 3: The physical prototype of the newly developed robot. The battery pack on top of the robot serves as a scale to show the robots' dimension.

The original system was designed to locate large quantity of robots wandering for survivors in rubbles and to feedback field data to the console for analyses. The search and rescue robots can be modified to collect field temperature, humidity and other useful data to support the camera system in determining whether there is a fire in the field. While the mobile robot is not the main focus of this research at its current state, the backbone of the static WSN is significant in adding reliability to the overall system. Without the mobile unit, all sensor nodes in the current system are set as reference nodes and can feedback temperature and humidity at designated interval to conserve power.

The sensor network has 9 modules programmed as reference nodes, and 2 modules programmed as blind nodes. The 9 reference nodes were distributed in the forest roughly resemble a square grid as shown in Figure 4 . The two blind nodes were installed on mobile modules. 


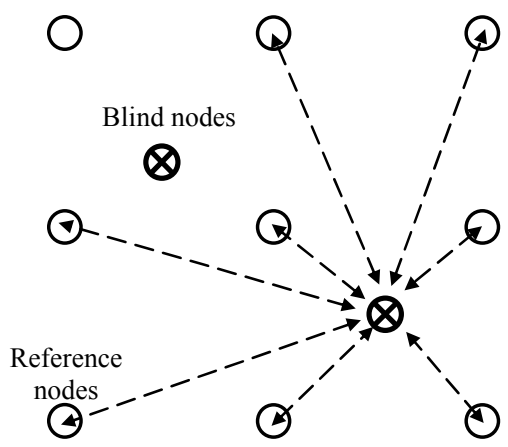

Figure 4: Zigbee modules in grid. Reference nodes are represented by blank circles, where crossed circles represent blind nodes.

Reference nodes are static nodes with known GPS positions and can assist other nodes to determine their locations. Blind nodes, on the other hand, are programmed to collect signals from all reference nodes to triangulate blind note positions. In the current forest fire detection system, only reference node is used. All reference nodes are equipped with temperature and humidity sensor. All reference node data are sent to the console via the nearest Internet connected WSN base station.

New blind nodes can be added anytime to expand the WSN. Algorithm used to estimate locations of the blind nodes within the sensor network is straightforward. To estimate its current location, the blind node on the mobile robot broadcast a specific signal to the surrounding. All reference nodes within range response to the signal by sending a packet containing the reference nodes' relative coordinate. The algorithm uses Received Signal Strength Indicator (RSSI) values to estimate distance from each reference node. Since RSSI value decreases as distance increases, the blind node would chose the 8 nearest reference nodes by comparing RSSI values between all reference nodes in range. Based on the strength of these returned signals and the origin of each signal included in the packet, position of the blind node can be estimated.

\section{Field test}

This research project was developed in partnership with the Guangdong Academy of Forestry since 2010. In 2011, the system successful completed a large-scaled field tests in HungShan, ZengCheng and QingYuan in the Guangdong Province (see Figure 5). Provincial Departments were involved to test the performance of the Computer Vision Wildfire Detection System. The system has met the requirement and is due to deploy into more cities in the Guangdong Province. 


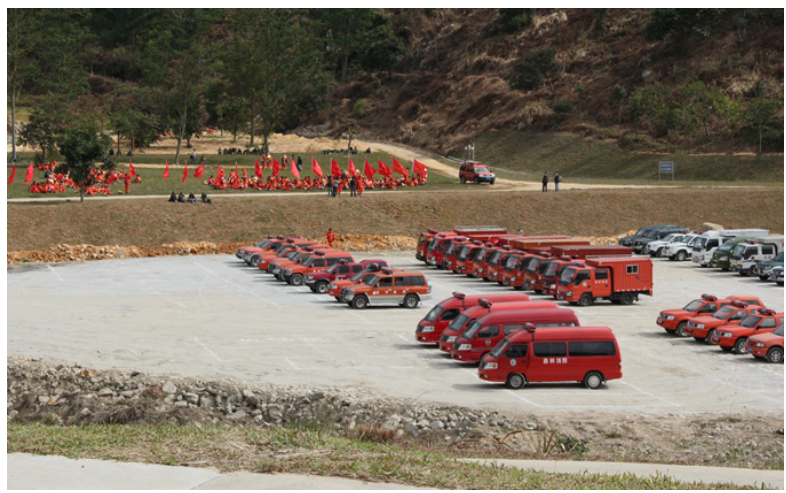

Figure 5: The largest field test in ZengCheng, Guangdong.

\subsection{Test condition}

A large-scale simulation exercise was conducted in ZengCheng, Guangdong Province, China in 2011 to demonstrate the system's accuracy and speed in detecting wildfire. Representatives from local and provincial departments of Guangdong Province attended the simulation exercise to witness how visual images and data fed back from the system at early stage of a fire can help fire officers to mitigate an emerging situation. The exercise also offered a realistic platform to demonstrate the system's intuitive display interface can help fire officers to digest ample amount of facts and figures in very short time. The intuitive display interface also provides a common ground for fire officers and provincial authorities in difference offices to discuss and coordinate response effort. A walk through of the simulation is illustrated below.

\subsubsection{Demonstration of computer interface at provincial level (Guangdong)}

The provincial forestry will see a map as shown below. The province is divided into grids (see Figure 6). The dimension of the grids can be modified to the user's preference. When a suspected fire is detected, the corresponding grid will change color to alert the fire officer.

\subsubsection{Demonstration of computer interface at field level (Guangdong)}

As shown in the map below, a fire is detected in ZengCheng causing the corresponding grid color to change to red (see Figure 6). The fire officer being alerted by the computer can navigate the computer pointer and click on the grid to obtain latest information of the emerging situation.

After clicking on the grid, the computer will display a 3D map of the area under examination along with images obtained from the thermal and video cameras. As shown in the screen captured image (Figure 7) the red circle led by a yellow line represents the location of the detected fire. Next to the red circle is a symbol of fire and strings of numerical values that describe latitude and longitude coordinate of the fire and the time it was first detected. The blue circle led by another yellow line represents the range that the cameras scanned. The 


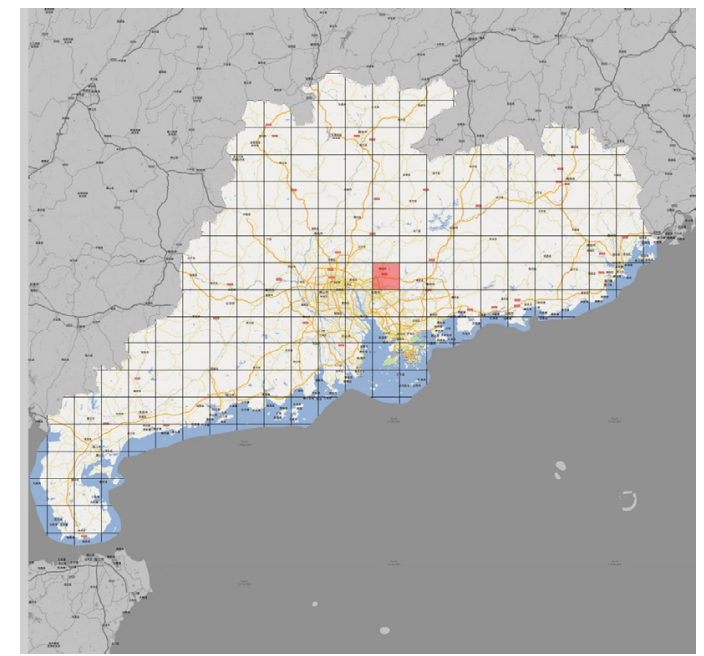

Figure 6: The grid color changes into red when suspected fire is detected.

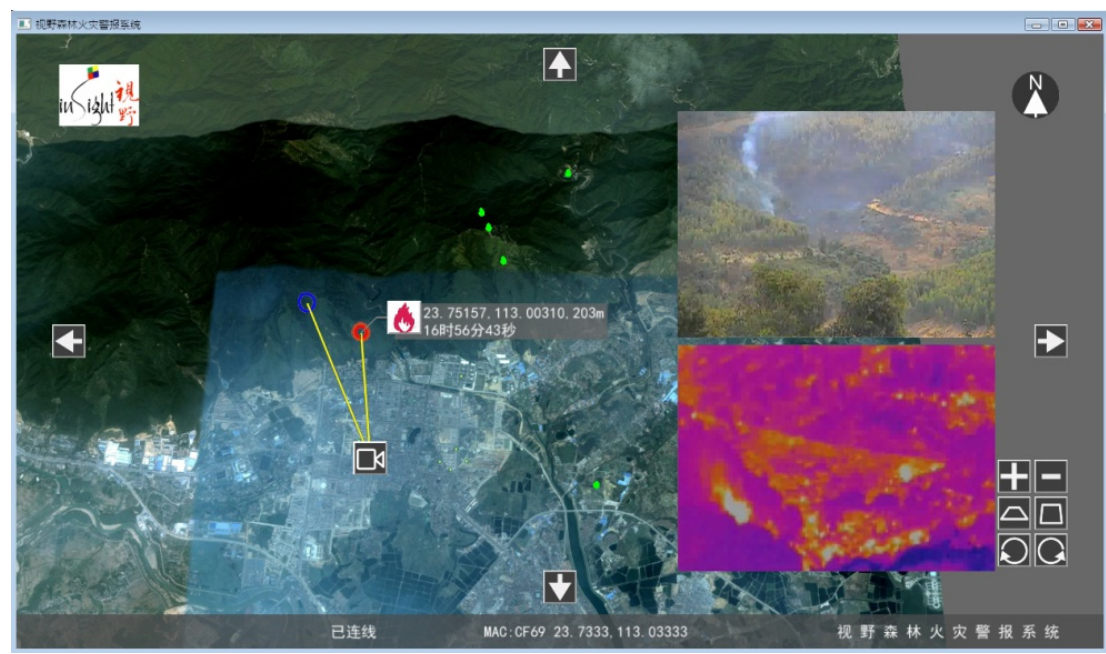

Figure 7: Screen captured from the computer user-interface.

square camera icon at where the two yellow lines met represents the location of the cameras where all the images were taken from. The two smaller images on the right are from video camera (top) and thermal camera (bottom).

\subsection{Remote PTZ manipulation}

To enable fire officers to obtain more information on the fire and surround situation, the Computer Vision Based Wildfire Detection System allows users to 
remotely control the PTZ camera by engaging the system in remote control mode. Priority to engage in remote control mode is given to fire officers at field level then to officers and provincial level. Whenever control conflict is detected the system will execute control command from field office and override all others. This mechanism is built on the assumption that field fire officers have better understanding of the location environment and fire prevention duty is rightly delegated to the field office. While this mechanism ensures frontline officers can obtain first hand information to made timely decision and report, provincial officers can rightly take control of the system when it is disengaged from the field office. Regardless of which office is controlling the system, images and data obtained from the system is shared to all authorized computer and mobile devices.

\section{Conclusion}

This paper described an intelligent wildfire detection system based on cameras and WSN. The combined use of vision and sensory data significantly improve system reliability and cost effectiveness. The camera system used infrared and daylight images to detect fire in all terrain visible to the camera, while the WSN returns critical data for monitoring valleys and other complex terrain that are costly to install additional cameras to monitor. The computer vision based wildfire detection system provided not only a way to lower cost and manpower but also a system that radically changes existing attitude in protecting invaluable resources that a sustainable planet cannot afford to lose. The computer vision system takes proactive stand in searching and identifying fire in areas not suitable to station human. Yet, it logs changes in the environment and evaluates fire by measuring rate of changes instead of following an absolute threshold. This is very similar to human's decision-making process. While the computer vision based system is currently designed for fire detection, it can also be applied to monitor illegal mining and logging by modifying the GSCF based detection algorithm and sensors in the system. By enhancing cost efficiency and performance in the fire detection system, we can save more forests and leave our future generations a planet they deserve.

\section{Acknowledgements}

This project benefited much from the strong technical and administrative support from the Guangdong Academy of Forestry, Ministry of Forestry of China, and INSIGHT Robotics (www.insightrobotics.com.). Through their assistance the system was able to perform realistic field test in Guangdong. The project also benefited from recognitions and awards from the industry. The Innovation and Technology awards for Asia Pacific region, 2011 APICTA ICT Awards and the Hong Kong ICT Awards all contributed to the financial side of the project. 


\section{References}

[1] UN News Centre. (2006, November 29 $9^{\text {th }}$ ). Rearing Cattle Produces More Greenhouse Gases than Driving Cars, UN Report Warns. Retrieved $6^{\text {th }}$ March, 2012, from the World Wide Web: http://www.un.org/apps/ news/story.asp?newsID $=20772 \& C R 1=$ warning

[2] Ko, A., Lau, H.Y.K., and Lau, T.L. (2005). "An Immuno Control Framework for Decentralized Mechatronic Control," International Journal of Unconventional Computing - 2005 Special Issues, pp. 225-280.

[3] de Castro, L.N. and Timmis, J. (2002). Artificial Immune Systems: A New Computational Intelligence Approach. New York: Springer-Verlag.

[4] Benjamini, E., Sunshine, G., and Leskowitz, S. (1996). Immunology: A Short Course. New York: Wiley-Liss.

[5] Wikipedia. (2012, March 2). International Year of Forests. Retrieved $6^{\text {th }}$ March, 2012, from the World Wide Web: http://en.wikipedia.org/ wiki/International_Year_of_Forests

[6] Swedberg, C. (2012). "RFID on Lookout for Wildfires in China," RFID Journal. Retrieved $6^{\text {th }}$ March, 2012, from the World Wide Web: http://www.rfidjournal.com/article/view/9218

[7] Aickelin, U., Bentley, P., Cayzer, S., Kim, J., and Mcleod, J., (2003). "Danger Theory: The Link between AIS and IDS," In Proceeding of 2nd International Conference on Artificial Immune Systems, (ICARIS 2003), pp. 147-155.

[8] Ko W.Y.A., Lau H.Y.K. and Lau T.L. (2004). "A decentralized control framework for modular robots," IEEE/RSJ International Conference on Intelligent Robots and Systems (IROS2004). Sendai, Japan, pp. 1774-1779.

[9] Ko, A., Lau, H.Y.K., and Lau, T.L, (2005) "SOHO security with mini selfbalancing robots", Industrial Robot: An International Journal, Vol. 32 Iss: 6, pp. $492-498$.

[10] Patel, S., Lorincz, K., Hughes, R., Huggins, N., Growdon, J.H., Welsh, M., and Bonato, P. (2007) "Analysis of Feature Space for Monitoring Persons with Parkinson's Disease With Application to a Wireless Wearable Sensor System," In Proceedings of the 29th IEEE EMBS Annual International Conference, Lyon, France, August 2007.

[11] Paek, J., Chintalapudi, K., Govindan, R., Caffrey, J., and Masri, S.(2005) "A Wireless Sensor Network for Structural Health Monitoring: Performance and Experience," Proc. $2^{\text {nd }}$ IEEE Workshop on Embedded Networked Sensors, IEEE CS Press, 2005.

[12] Werner-Allen, G., Lorincz, K., and Welsh, M. (2006) "Deploying a Wireless Sensor Network on an Active Volcano," IEEE Internet Computing, March issue, pp. 18-25.E. Benjamini, G. Sunshine, S. Leskowitz. Immunology: A Short Course. Wiley-Liss, New York, USA, 1996. 